\title{
Compact High-Brightness Soft X-Ray Cherenkov Sources
}

\author{
Walter Knulst, Jom Luiten, and Jan Verhoeven
}

\begin{abstract}
Compact narrow-band high-brightness soft X-ray sources based on the Cherenkov effect are very promising. We discuss the theoretical basis for this novel electron-accelerator-based source. We present results of experiments, which confirm the theoretical expectations and demonstrate that intense narrow-band Cherenkov light can be produced at the silicon L-edge $(99.7 \mathrm{eV})$ and, in the water window, at the titanium L-edge $(453 \mathrm{eV})$ and the vanadium $L$-edge $(512 \mathrm{eV})$. On the basis of theory and experiment, we show that a compact high-brightness Cherenkov source may be realized, which fulfills the requirements for practical soft X-ray microscopy and photoelectron spectroscopy.
\end{abstract}

Index Terms-Cherenkov effect, extreme ultraviolet (EUV), photoelectron spectroscopy, soft X-rays, X-ray microscopy.

\section{INTRODUCTION}

$\mathbf{P}$ RESENTLY, the soft X-ray sources with the highest brightness (number of photons per second, per unit area, per unit solid angle, and per unit relative bandwidth) are those based on undulators in storage rings. Many powerful soft X-ray techniques which require high brightness, such as $\mathrm{X}$-ray microscopy, X-ray crystallography, X-ray diffraction, and fluorescence X-ray microprobes, are therefore limited to these synchrotron light facilities. However, several of these applications require substantially less than the brightness levels offered by undulator radiation, so with further development of alternative sources they may become available to smaller institutes as well. This possibility has sparked a great deal of interest in compact (laboratory-sized) high-brightness soft $\mathrm{X}$-ray sources [1].

The most well-known compact X-ray source is the X-ray tube, which has been used in many fields of research ever since the discovery of X-rays by Röntgen. However, the emission intensity of fluorescence radiation in the soft $X$-ray region, which requires low- $\mathrm{Z}$ materials, is very low, making the soft $\mathrm{X}$-ray

Manuscript received May 4, 2004; revised August 24, 2004. This work was supported by the Technology Foundation STW, applied science division of NWO, and the technology programme of the Ministry of Economic Affairs, The Netherlands.

W. Knulst is with Delft University of Technology, 2600 AA Delft, The Netherlands.

J. Luiten is with Eindhoven University of Technology, 5600 MB Eindhoven, The Netherlands.

J. Verhoeven is with the FOM Institute for Atomic and Molecular Physics, 1098 SJ Amsterdam, The Netherlands.

Digital Object Identifier 10.1109/JSTQE.2004.837738 tube generally not very attractive as a source. Recent developments in this direction are, for instance, an extreme ultraviolet (EUV) tube producing silicon L-band fluorescence radiation (centered at $92 \mathrm{eV}$ ) [2] and a liquid-water-anode producing oxygen $\mathrm{K}_{\alpha}$-fluorescence radiation $(525 \mathrm{eV})$ [3].

More intense, but still compact, alternatives are plasma-based sources, which cover the range from visible radiation up to $\mathrm{X}$-rays of a few kiloelectronvolts. Over the last decade, the efficiency of these soft X-ray sources has improved enormously. Plasma-based sources can be divided into pinch plasma (PP) and laser-produced plasmas (LPP). PP sources, in which a high current pulse is sent through a plasma to make it collapse, are presently the only sources capable of producing the average power levels at $13.5 \mathrm{~nm}$ required for industrial high-throughput EUV lithography. In LPP sources nanosecond laser pulses are used to create plasmas, emitting line spectra from highly ionized atoms such as $\mathrm{C}, \mathrm{N}, \mathrm{O}$, or Xe. As a side effect, these sources produce a certain amount of debris, which can be reduced by using gaseous or liquid targets [4], [5]. Recently, it has been shown that LPP sources are sufficiently bright for practical imaging in the water window [8].

A relatively recent development is the high-harmonic (HH) source: when a very short, highly intense laser pulse is sent through a gas, very high odd harmonics of the frequency of the incident laser pulse are generated by nonlinear interaction [6]. The conversion efficiency to high photon energies, however, is generally limited by the lack of phase matching. Recently, proper phase matching has been extended into the water-window region [7]. HH sources operating at $13 \mathrm{~nm}$ are presently sufficiently bright for practical imaging [9].

Compact electron-accelerator-based sources [10] have received relatively little attention until now. Among the interactions of relativistic electrons with a medium that cause emission of radiation, such as transition radiation (TR), channeling radiation and parametric X-rays, especially Cherenkov radiation (CR), is a promising candidate for a compact soft $\mathrm{X}$-ray source. As we will show in this paper, in the soft X-ray region the Cherenkov radiation is characterized by a single-line spectrum and by forwardly directed emission and only requires low-relativistic electrons from a laboratory-sized accelerator.

Cherenkov radiation is emitted by a charged particle if its velocity $(v)$ exceeds the phase velocity of light in a medium $\left(v_{\phi}=c / n\right)$ and is therefore limited to the wavelength regions where the real part of the refractive index exceeds unity $(n>1)$. 
Cherenkov radiation is emitted at an angle with respect to the electron trajectory which is given by

$$
\cos \theta=\frac{v_{\phi}}{v}=\frac{c}{n v}
$$

In the visible region Cherenkov radiation is a well-known effect, which is often applied in high-energy particle detection [14]. In the soft X-ray region, however, the refractive index is generally smaller than unity and materials are highly absorbing. Therefore, Cherenkov radiation in the soft X-ray region was excluded for a long time. In 1981, Bazylev et al. [11] realized, however, that at some inner-shell absorption edges the refractive index exceeds unity, implying that Cherenkov radiation can be generated in a narrow-band region around certain absorption edges. They demonstrated this experimentally for the carbon K-edge (284 eV), using 1.2-GeV electrons. Later, Moran et al. [15] showed that Cherenkov radiation is emitted by $75-\mathrm{MeV}$ electrons in silicon at the L-edge $(99.7 \mathrm{eV})$ and in carbon at the K-edge. Recently, we have shown that Cherenkov radiation at the silicon L-edge can be generated with 5-MeV electrons [12] and at the titanium and vanadium L-edge with $10-\mathrm{MeV}$ electrons [13]. It turns out that the potential brightness of this new type of compact source is sufficient for imaging applications. In this paper, the theoretical and experimental aspects of generating soft X-ray Cherenkov radiation and its potential as a high-brightness source are treated in somewhat more detail than in the first brief reports [12], [13].

This paper is structured as follows: In Section II, the theory of soft X-ray Cherenkov radiation is presented. Although the electromagnetic theory of Cherenkov radiation was formulated already a long time ago by Frank and Tamm [19], this theory does not apply anymore in the soft X-ray regime because of the strong absorption of materials. Instead, a description is required in terms of the Ginzburg-Frank equation, which was originally developed for transition radiation [20], [21]. In Sections III-V, the measurements of soft X-ray Cherenkov radiation are presented. The main purpose of the experiments is to demonstrate that moderate electron energies are already sufficient to generate narrow-band soft X-ray Cherenkov radiation in different materials. We have firmly established the generation of silicon L-edge Cherenkov radiation with 5-MeV electrons (Section III) and titanium $\mathrm{L}$-edge and vanadium $\mathrm{L}$-edge Cherenkov radiation with $10-\mathrm{MeV}$ electrons (Section IV). In addition, some attempts have been made to establish Cherenkov radiation at the carbon-K edge and the nickel-L edge (Section V). The fact that we did not observe Cherenkov radiation using carbon puts a new upper limit on the value of the refractive index of carbon at the $\mathrm{K}$-edge. On the basis of the measured soft X-ray Cherenkov spectra, the achievable brightness is discussed in Section VI for the case of an optimum electron beam. The main brightness limiting factor turns out to be the maximum heat load on the foil. We find that a Cherenkov source in the water-window spectral region potentially has a brightness comparable to the best laser-produced plasma source. We, therefore, conclude that the proposed soft X-ray Cherenkov source fulfills the requirements of practical soft X-ray microscopy and X-ray photoelectron microscopy.

\section{THEORY OF SOFT X-RAY CHERENKOV RADIATION}

\section{A. Ginzburg-Frank Theory}

When a relativistic electron is sent through a foil, several radiation phenomena occur. Due to the fact that the Cherenkov radiation is generated in a finite solid-state medium, it is always accompanied by transition radiation, which is generated by the electron at the medium-vacuum interfaces. Therefore, this effect has to be taken into account as well. All other radiation phenomena, such as fluorescence radiation, Bremsstrahlung, and visible transition radiation are discussed in the context of the experimental results in Sections III-V.

Until now, only a few theoretical studies [16], [17] have addressed the possibility of generating soft X-ray Cherenkov radiation at inner-shell photon energies. We start the analysis from the Ginzburg-Frank equation [20], [21], [18], which was initially intended to describe transition radiation only. As this equation is the exact solution of Maxwell's equations in a system consisting of two adjacent semi-infinite dielectric media, it automatically describes Cherenkov radiation as well.

Suppose an electron moves through the interface between a medium with dielectric constant $\epsilon$ and vacuum. The number of photons $N$ emitted into the vacuum per electron, per unit bandwidth, and per unit solid angle, the so-called spectral angular yield, is given by [18], [20], and [21]

$$
\begin{aligned}
\frac{d^{2} N}{d \omega d \Omega}=\frac{\alpha}{\omega} \frac{\beta^{2}}{\pi^{2}} & \frac{|\epsilon-1|^{2} \sin ^{2} \theta \cos ^{2} \theta}{\left|\epsilon \cos \theta+\sqrt{\epsilon-\sin ^{2} \theta}\right|^{2}} \\
& \times \frac{\left|1-\beta^{2}-\beta \sqrt{\epsilon-\sin ^{2} \theta}\right|^{2}}{\left|\left(1-\beta^{2} \cos ^{2} \theta\right)\left(1-\beta \sqrt{\epsilon-\sin ^{2} \theta}\right)\right|^{2}}
\end{aligned}
$$

with $\beta=v / c$ the relative electron velocity and $\alpha$ the fine structure constant. Using Snell's law for a nonabsorbing medium $(n=\sqrt{\epsilon})$, it is easy to see that the factor $\left(1-\beta \sqrt{\epsilon-\sin ^{2} \theta}\right)$ in the denominator becomes zero and, thus, the spectral angular yield is infinite at exactly at the Cherenkov angle [see (1)]. This is due to the fact that in the case of a semi-infinite medium Cherenkov radiation is generated over an infinite path length. However, in reality, either the path length is finite or the medium is absorbing, making the Cherenkov yield finite. In the soft X-ray regime all media are strongly absorbing, which is taken into account by a complex-valued dielectric constant: $\epsilon=1+\chi=1+\chi^{\prime}+i \cdot \chi^{\prime \prime}$. In addition, in this regime the absolute value of the dielectric constant is always close to unity, i.e., $|\chi| \ll 1$. The absorption length is then given by $c / \omega \chi^{\prime \prime}$, which typically is less than $1 \mu \mathrm{m}$ and therefore much smaller than the thickness of the foils we used. In this paper, we therefore approximate the foils by semi-infinite absorbing media, which allows us to use the Ginzburg-Frank equation (2) with a complex-valued dielectric constant.

We may simplify (2) by only considering ultra-relativistic electrons (Lorentz factor $\gamma \equiv 1 / \sqrt{1-\beta^{2}} \gg 1$ ) and therefore only small emission angles $(\theta \ll 1)[21]$

$$
\frac{d^{2} N}{d \omega d \Omega}=\frac{\alpha}{\omega} \frac{\theta^{2}}{\pi^{2}}\left|\frac{1}{-\gamma^{-2}-\theta^{2}}-\frac{1}{\chi-\gamma^{-2}-\theta^{2}}\right|^{2} .
$$

Note that in the approximation that $\gamma \gg 1$ and $|\chi| \ll 1$, the Cherenkov angle is simply given by

$$
\theta \approx \sqrt{\chi^{\prime}-\gamma^{-2}}
$$



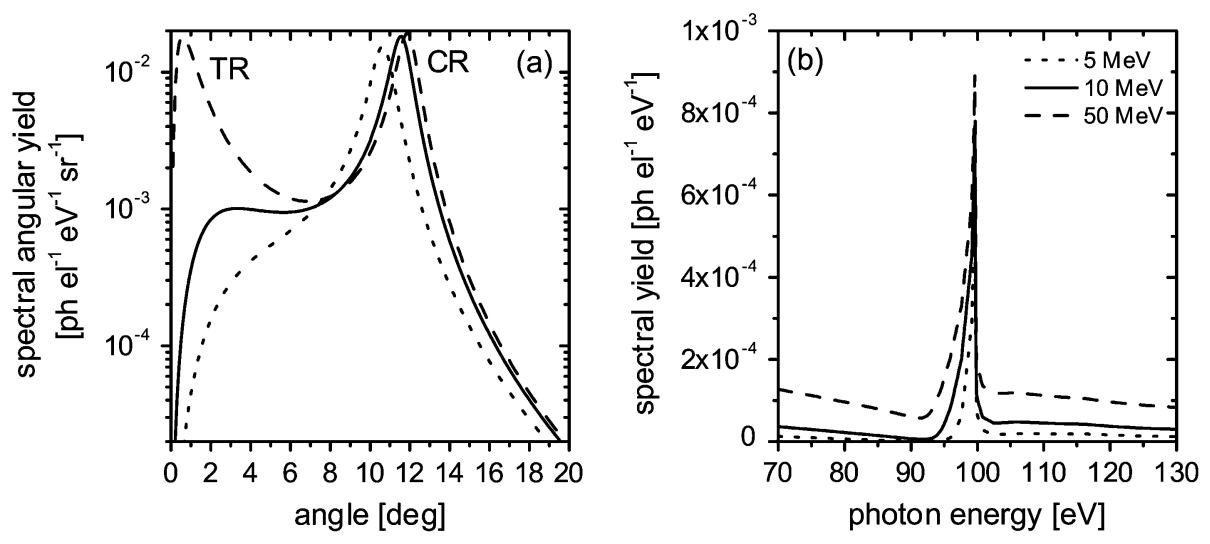

Fig. 1. (a) Spectral angular yield for silicon at $99.7 \mathrm{eV}$ for different electron energies: $5 \mathrm{MeV}$ (dotted curve), $10 \mathrm{MeV}$ (solid curve), and $50 \mathrm{MeV}$ (dashed curve). (b) Spectral yield calculated by (5) for different electron energies, namely 5, 10, and $50 \mathrm{MeV}$.

Equation (3) is usually applied in the X-ray region to calculate the transition radiation yield. Generally, a simple plasma-frequency model of the dielectric constant is substituted for the susceptibility [18], [21], which precludes the possibility of generating Cherenkov radiation. Such an approach is, in fact, only correct far from atomic resonances. To calculate the transition and Cherenkov radiation at regions of anomalous dispersion, the full frequency-dependent complex dielectric constant has to be substituted into (3). The solid curve in Fig. 1(a) shows the result of such a calculation for the case of silicon at a photon energy of $99.7 \mathrm{eV}$, using $10-\mathrm{MeV}$ electrons. Clearly, a large peak is visible that is associated with silicon L-edge Cherenkov radiation. The angle of the Cherenkov maximum agrees with the approximated Cherenkov angular relation (4). At smaller angles a secondary maximum is barely visible that can be identified as transition radiation.

Strictly speaking, it is not possible to separate mathematically the Cherenkov spectral angular yield from the transition radiation spectral angular yield in (3); the two phenomena are intertwined as both of them originate from a single process. However, the angular radiation profile is generally characterized by two angles of maximum intensity, each of which behaves differently as a function of electron energy. One of these peaks is usually associated with transition radiation and the other with Cherenkov radiation, as if they are independent phenomena. For increasing electron energy the transition radiation peaks at smaller angles, i.e., $\theta_{\mathrm{TR}} \approx \gamma^{-1}$, and the spectral angular yield increases. The Cherenkov angle, on the other hand, approaches a constant value for increasing electron energy. If the electron energy is such that the angle of maximum transition radiation intensity and the Cherenkov angle coincide, then the two radiation phenomena are truly indistinguishable.

By integrating (3) over all emission angles, one finds in the approximation that $\chi^{\prime}-\gamma^{-2}<<\chi^{\prime \prime}$ in the following expression for the spectral yield [11]:

$$
\begin{aligned}
& \frac{d N}{d \omega}=\frac{\alpha}{\pi \omega}\left\{\left(\frac{1}{2}-\frac{\gamma^{-2}}{\chi^{\prime}}\right) \ln \right.\left.\left(\frac{\left(\chi^{\prime}-\gamma^{-2}\right)^{2}}{\gamma^{-4}}\right)-1\right\} \\
&+\frac{\alpha}{\omega} \frac{\chi^{\prime}-\gamma^{-2}}{\chi^{\prime \prime}} \eta\left(\chi^{\prime}-\gamma^{-2}\right)
\end{aligned}
$$

with $\eta$ the Heaviside step function. Now the contributions due to Cherenkov radiation and transition radiation can be separated; the second part on the right-hand side is equal to the soft X-ray Cherenkov spectral yield given by Frank-Tamm theory [22], if absorption is taken into account in a phenomenological way [12]. For highly relativistic electrons $\left(\gamma^{-2} \ll \chi^{\prime} \ll 1\right)$ (5) reduces to

$$
\frac{d N}{d \omega}=\frac{\alpha}{\pi \omega} \ln \left(\gamma^{2} \chi^{\prime}\right)+\frac{\alpha}{\omega} \frac{\chi^{\prime}}{\chi^{\prime \prime}} \eta\left(\chi^{\prime}\right)
$$

Equation (6) shows that the Cherenkov radiation yield becomes constant in the high-energy limit, while the transition radiation part still increases slowly with the logarithm of $\gamma$. For moderate electron energies (up to $25 \mathrm{MeV}$ ), however, the transition radiation spectral yield can be neglected with respect to the Cherenkov spectral yield as is shown in Fig. 1(b) for the case of silicon using 5- and $10-\mathrm{MeV}$ electrons. Only for $50-\mathrm{MeV}$ electrons does the transition radiation spectral angular yield become approximately equal to the Cherenkov spectral angular yield [see Fig. 1(a)]. Even then, the Cherenkov spectral yield is still much higher than the transition radiation spectral yield [see Fig. 1(b)]. The reason is that Cherenkov radiation is emitted at larger angles and, therefore, in a larger total solid angle.

\section{B. Soft X-Ray Cherenkov Radiation Characteristics}

We will now discuss the characteristics of Cherenkov radiation in the soft X-ray region on the basis of the theory discussed in the previous section.

1) The Cherenkov radiation is characterized by a narrow bandwidth because the real part of the susceptibility $\chi^{\prime}$ exceeds unity only in a narrow spectral region. The step in the imaginary part of the susceptibility $\chi^{\prime \prime}$ narrows the Cherenkov spectrum even further, mainly to the part at the low photon-energy side of the edge. Typically, the Cherenkov spectrum has a full-width at half-maximum (FWHM) of a few electron volts.

2) Equation (4) shows that the maximum emission angle is given by $\theta_{\max }=\sqrt{\chi_{\max }^{\prime}}$, which is typically a few degrees. The Cherenkov radiation is therefore emitted in a narrow forwardly directed angular profile. This angular profile is a hollow cone because the spectral Cherenkov intensity (5) is proportional to the emission angle squared and therefore zero for $\theta=0$.

3) The Cherenkov condition $\chi^{\prime}-\gamma^{-2}>0$ in (5) shows that the minimal Lorentz factor required is $\gamma_{\min }=1 / \sqrt{\chi_{\max }^{\prime}}$, 
TABLE I

Properties of SuItABle CHERENKOV RADIATION EMITTERS IN THE WATER-WiNDOW REgION

\begin{tabular}{ccc|cc|c|c|c|c|c}
\hline $\mathrm{Z}$ & Element & Edge & $\begin{array}{c}E_{p h}=\hbar \omega \\
{[\mathrm{eV}]}\end{array}$ & $\begin{array}{c}\Delta E \\
{[\mathrm{eV}]}\end{array}$ & $\begin{array}{c}\rho \\
{\left[\mathrm{g} / \mathrm{cm}^{3}\right]}\end{array}$ & $\chi_{\max }^{\prime}$ & $\begin{array}{c}E_{\min } \\
{[\mathrm{MeV}]}\end{array}$ & $\begin{array}{c}\theta_{\max } \\
{[\mathrm{deg}]}\end{array}$ & $\begin{array}{c}\text { Yield } \\
{[\mathrm{ph} / \mathrm{el}]}\end{array}$ \\
\hline 19 & $\mathrm{~K}$ & $\mathrm{~L}_{\mathrm{III}}$ & 294.6 & 1.2 & 0.862 & $4.31 \times 10^{-3}$ & 7.3 & 2.5 & $2.0 \times 10^{-4}$ \\
20 & $\mathrm{Ca}$ & $\mathrm{L}_{\text {III }}$ & 346.2 & 1.6 & 1.55 & $4.92 \times 10^{-3}$ & 6.8 & 2.9 & $2.0 \times 10^{-4}$ \\
21 & $\mathrm{Sc}$ & $\mathrm{L}_{\text {III }}$ & 398.7 & 1.9 & 2.989 & $6.81 \times 10^{-3}$ & 5.7 & 3.8 & $2.6 \times 10^{-4}$ \\
22 & $\mathrm{Ti}$ & $\mathrm{L}_{\text {III }}$ & 453.8 & 2.0 & 4.54 & $7.01 \times 10^{-3}$ & 5.5 & 3.9 & $2.4 \times 10^{-4}$ \\
23 & $\mathrm{~V}$ & $\mathrm{~L}_{\text {III }}$ & 512.1 & 1.2 & 6.11 & $6.86 \times 10^{-3}$ & 5.7 & 3.8 & $1.2 \times 10^{-4}$ \\
\hline
\end{tabular}

which is typically in the range between 10 and 50, i.e., electron energies ranging from 5 to $25 \mathrm{MeV}$. Such electron energies can be generated with compact accelerators. Much higher electron energies are not necessary, because the Cherenkov spectral yield saturates for electron energies corresponding to a few times $\gamma_{\mathrm{min}}$.

\section{EXPERIMENTAL RESUlts: SilicON L-EDGE CHERENKOV RADIATION}

Soft X-ray Cherenkov radiation at the silicon L-edge $(99.7 \mathrm{eV})$ can be generated in a silicon foil. Before the authors of this paper, only Moran et al. [15] had observed Si-L Cherenkov radiation, which they produced by sending $75-\mathrm{MeV}$ electrons at grazing incidence through a foil. The intention of our experiments was to show that $5-\mathrm{MeV}$ electrons are already sufficient to generate $\mathrm{Si}-\mathrm{L}$ Cherenkov radiation with a reasonable yield. Our first results on Si-L Cherenkov radiation generated by $5-\mathrm{MeV}$ electrons have been reported by Knulst $e t$ al. [12]. Improved measurements of Si-L Cherenkov radiation, which were performed at a later stage, are discussed in detail in [23].

These measurements constitute the first detailed spectrally and angularly resolved observation of Si-L Cherenkov radiation. The radiation was analyzed using a crude spectrometer based on a Si-Mo-multilayer mirror and an absolute XUV-photodiode placed in a $\theta-2 \theta$ configuration [12]. Our measurements show that on the basis of the silicon refractive index data of Henke database [24], the measured Cherenkov radiation can be accurately predicted. The measured yield is twice as low as the theoretically predicted value of $8 \times 10^{-4}$ photon/electron. This discrepancy can be attributed to the combination of electron scattering in the silicon foil and remaining systematic errors. In this way, we demonstrated for the first time that soft X-ray Cherenkov radiation can be generated with moderate electron energies. The resulting soft X-ray brightness and implications for source development are discussed in Section VI-B.

\section{EXPERIMENTAL RESULTS: TITANIUM L-EDGE AND VANADIUM L-EDGE CHERENKOV RADIATION}

\section{A. Cherenkov Radiation in the Water Window}

In Table I, several materials are listed that, theoretically, should emit Cherenkov radiation in the water window. All materials emit Cherenkov radiation with a typical FWHM spectral width of about $1.5 \mathrm{eV}$ and a yield of a few times $10^{-4}$ photon per electron. These calculations are based on very few data

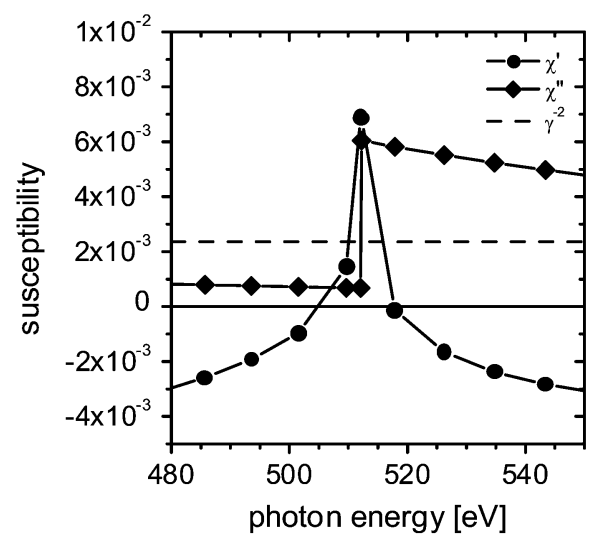

Fig. 2. Susceptibility $\chi$ of vanadium around the L-edge (512 eV). Dashed line indicates the Cherenkov threshold for $10-\mathrm{MeV}$ electrons.

points of the refractive index, as is illustrated in Fig. 2 by the susceptibility of vanadium around the L-edge $(512 \mathrm{eV})$. For our measurements, we selected titanium $(453 \mathrm{eV})$ and vanadium $(512 \mathrm{eV})$, because both these metals are readily available in $\mu$ m-thick foils.

\section{B. Experimental Setup}

For the measurements at photon energies corresponding to the water window or higher, a different detection setup has been used than for silicon L-edge Cherenkov radiation. The detector unit is a dedicated soft X-ray charge-coupled device (CCD) camera that has been developed by Space Research Organization, The Netherlands, for X-ray astronomy. The CCD chip is a copy of the chips that are used in the European XMM-Newton satellite [25]. The camera is capable of measuring the energy of individual photons with a high detection efficiency $(\approx 90 \%)$. Photon energies between $280 \mathrm{eV}$ and $15 \mathrm{keV}$ can be spectrally resolved. The FWHM-spectral resolution is $165 \mathrm{eV}$ at $1.5 \mathrm{keV}$ and scales with the square root of the photon energy. The CCD chip is cooled to $-80^{\circ} \mathrm{C}$ to reduce the dark current. During the experiments the $\mathrm{CCD}$ camera is operated in the photon counting mode in which only $10 \%$ of the 800000 pixels may be illuminated at any time to enable the event reconstruction algorithm. The average accelerator beam current was therefore set to a very low value of about $10^{9}$ electrons per second. In front of the CCD chip, a thin aluminum-carbon filter (layer thicknesses of 150 and $27 \mathrm{~nm}$, respectively) with a diameter of $16 \mathrm{~mm}$ was mounted to block any visible transition radiation. This filter limits the total detection solid angle to $2.2 \times 10^{-4} \mathrm{sr}$. 
The experimental setup was designed to analyze the emitted radiation as a function of emission angle. Because the emitted Cherenkov radiation has a cone-like intensity profile that is cylindrical symmetric around the electron trajectory, it is sufficient to measure the profile in one plane by moving the CCD camera along an arc [23].

The electrons are generated by a $10-\mathrm{MeV}$ traveling wave linear accelerator (LINAC), which was part of a former medical radiation treatment machine (M.E.L. SL-75). This electron accelerator produces 9- to $11-\mathrm{MeV}$ electrons, depending on the accelerator current, with a typical energy spread of $4 \%$ and a transverse emittance (i.e., root mean square (rms) spot size times rms divergence) of about $10 \mathrm{~mm} \cdot \mathrm{mrad}$. After the electrons have passed through the Cherenkov foil, a $90^{\circ}$ dipole magnet bends the electron beam into a beam dump where the accelerator current can be measured.

We have used $10-\mu \mathrm{m}$-thick $25 \times 25 \mathrm{~mm}^{2}$ titanium and vanadium foils, which have been purchased at Goodfellow.

\section{Ti-L and V-L Cherenkov Spectra}

The results of the measurements on titanium and vanadium presented in this section have been reported briefly before by Knulst et al. [13].

We discuss the vanadium measurements only, because the titanium spectra are very similar. In Fig. 3, three spectra obtained at different detection angles are plotted after event reconstruction has been performed. In these spectra, a few radiation phenomena can be observed caused by the passage of the electron beam through the foil. The strong peaks in Fig. 3 can be identified as Cherenkov radiation (CR). The offset under the Cherenkov peak is a low-intensity continuous background of transition radiation (TR) that decreases with photon energy and has its maximum intensity at an emission angle of $\gamma^{-1}$, i.e., $2.8^{\circ}$ for $10-\mathrm{MeV}$ electrons. The narrow peak on the left side of the Cherenkov peak is an artefact that always appears after performing event reconstruction. At higher photon energies, just outside the plotted range of Fig. 3, a weaker peak appears, which can be identified as fluorescence radiation. The presence of a very small background around the fluorescence peak is caused by Bremsstrahlung, which consists mainly of hard X-rays. Bremsstrahlung is emitted within an angle of approximately $\gamma^{-1}$. By changing the detection angle the relative intensities of these radiation phenomena can be varied. At large detection angles $\left(\Theta=9^{\circ}\right)$ the fluorescence radiation, which is emitted isotropically over $4 \pi \mathrm{sr}$, is dominant, because all other radiation phenomena are emitted in a narrow cone around the electron beam. This fluorescence radiation is used for the energy calibration of the CCD camera. At smaller detection angles (e.g., $\Theta=4^{\circ}$ and $\Theta=0^{\circ}$ ) Cherenkov radiation, transition radiation, and Bremsstrahlung are dominant.

The FWHM width of the measured vanadium peak is $102 \mathrm{eV}$, which is completely determined by the spectral resolution of the CCD camera. The measured peak can therefore be fitted by a Gaussian profile with a linear offset. Based on the peak positions obtained from the Gaussian fit, the vanadium Cherenkov peak is experimentally determined at $519 \pm 3 \mathrm{eV}$ and using the same
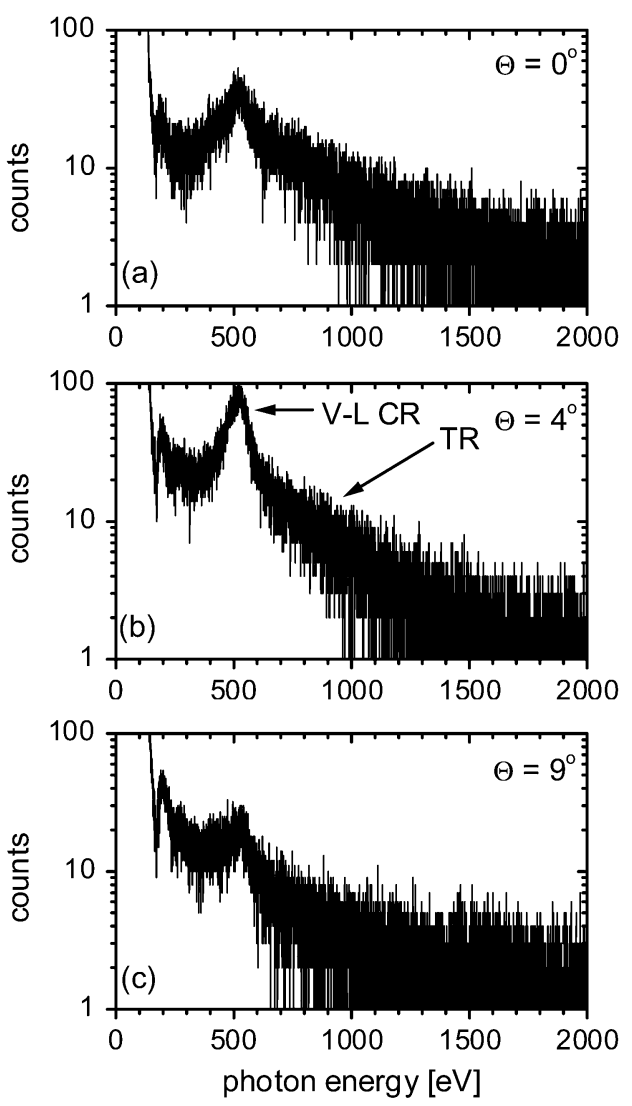

Fig. 3. CCD pulse height spectrum after event reconstruction obtained using a vanadium foil measured at detection angles: (a) $\Theta=0^{\circ}$, (b) $\Theta=4^{\circ}$, and (c) $\Theta=9^{\circ}$.

analysis the titanium peak is determined at $459 \pm 2 \mathrm{eV}$. A small, but significant, difference is observed with the theoretical peak positions at their respective L-edges, i.e., 512 and $453 \mathrm{eV}$. The fact that we measure the peaks at slightly higher photon energies is explained in the following.

In Fig. 4(a), the theoretical spectral yield (dotted line) for vanadium is plotted, which is calculated using the Ginzburg-Frank equation (2) and integrated over the solid angle of the detector around an emission angle of $4^{\circ}$. In this spectrum (dotted curve), clearly the high Cherenkov peak is visible on a low-intensity background of transition radiation, which decreases with photon energy. By multiplying this spectrum with the transmission of the $\mathrm{Al} / \mathrm{C}$-filter the spectrum can be calculated of the photon flux incident on the CCD chip (dashed curve). Then, by convolving this spectrum with the spectral response of the CCD camera, i.e., a Gaussian profile with a FWHM width of $102 \mathrm{eV}$, the expected CCD-camera spectrum is calculated (solid curve). The peak position of the calculated CCD-camera spectrum lies at $522 \mathrm{eV}$ for vanadium. The measured peak position of vanadium agrees within the experimental error with the theoretical value obtained from this analysis. Using the same analysis, we find for titanium a calculated position of $458 \mathrm{eV}$ that also agrees within the experimental error with the measured value. These shifts are due to the fact that at a photon energy a few electron volts below the L-edges the real part of the susceptibility goes through zero and therefore at this specific photon energy both transition radiation 

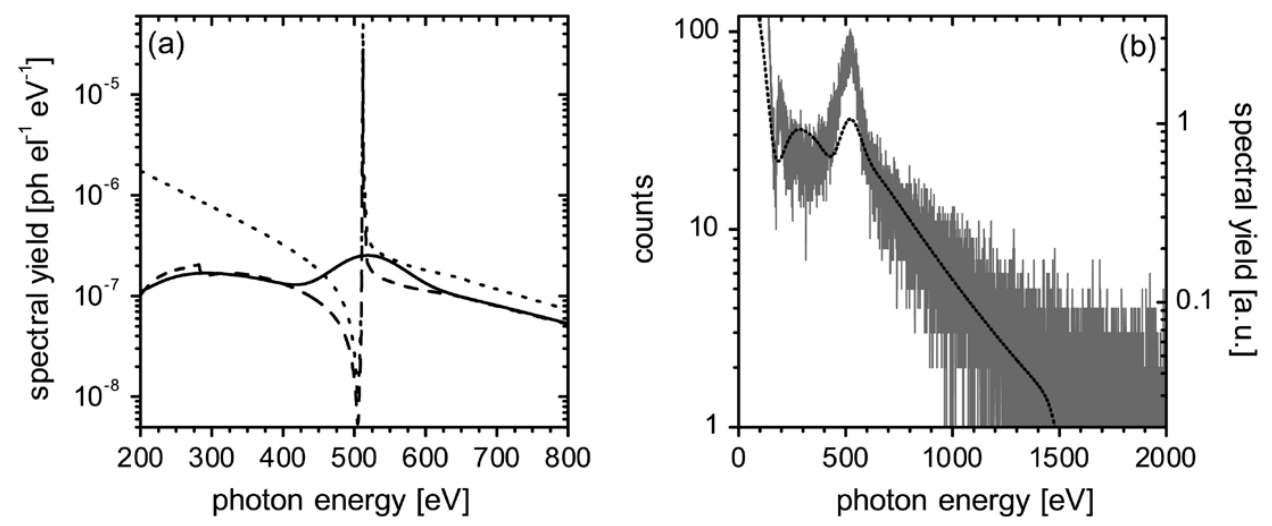

Fig. 4. (a) Theoretical calculation of the expected CCD spectrum consisting of the theoretical spectral yield (dotted curve), which is multiplied with the filter transmission (dashed curve), and finally convolved with the CCD spectral response (solid curve) for vanadium. (b) Pulse height spectrum of the radiation produced by $10-\mathrm{MeV}$ electrons in vanadium at $\Theta=4^{\circ}$. Dashed curve is the calculated CCD spectrum from (a).
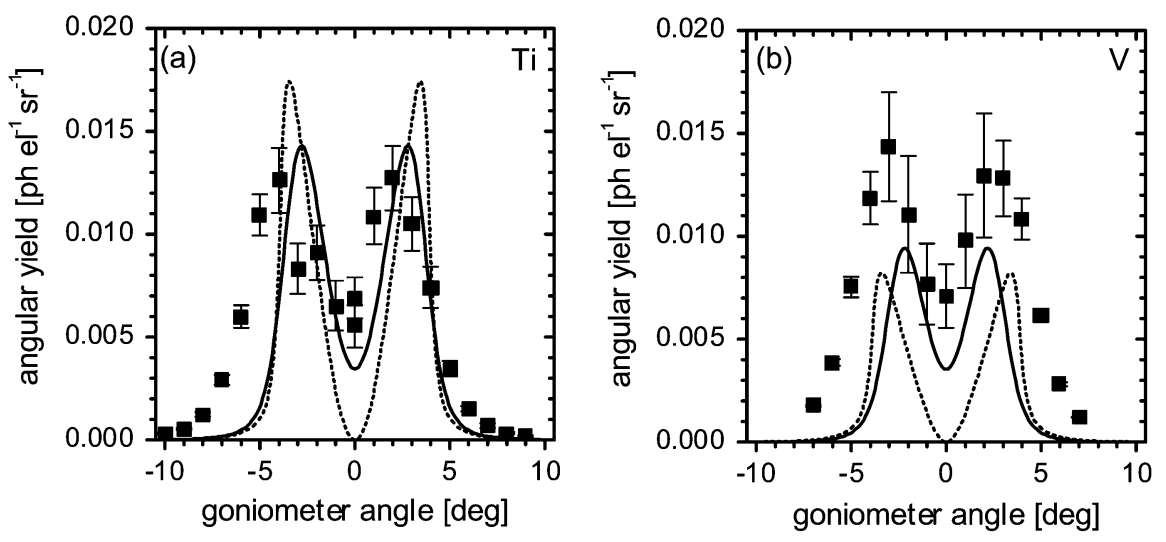

Fig. 5. Measured Cherenkov angular yield as a function of detection angle generated by 10-MeV electrons in (a) titanium and (b) vanadium. Dotted curves are theoretical angular Cherenkov yields for an ideal parallel beam. Solid curves take elastic scattering in the foil into account.

and Cherenkov radiation are absent. The resulting dip shifts the peaks to slightly higher energies. In Fig. 4(b), the calculated CCD camera spectrum for vanadium is plotted together with the measured spectrum at $\Theta=4^{\circ}$, showing that the low-energy part of the measured spectra is completely described in terms of Cherenkov and transition radiation. The measured Cherenkov peak for the case of vanadium is higher than the theoretical curve, which will be discussed below.

It is important to realize that the theoretical FWHM spectral width of Cherenkov radiation is about $2 \mathrm{eV}$ for both titanium and vanadium. Note that the actual height of the Cherenkov peaks is therefore approximately 50 times higher than the measured peaks in the spectra, which can only be observed by using a high-resolution spectrometer. This means that the radiation spectra in the soft X-ray region of both titanium and vanadium are completely dominated by a single intense peak of Cherenkov radiation.

\section{Ti-L and $V$ - $L$ Cherenkov Angular Profile and Total Yield}

Similar spectra as in Fig. 3 have been obtained at detection angles ranging from $\Theta=-10^{\circ}$ to $+10^{\circ}$ with steps of $1^{\circ}$ for both titanium and vanadium. From each spectrum the area of the Cherenkov peak is determined, which gives the number of photons at each detector position. Then, by taking into account the detection efficiency of the CCD camera, the transmission of the $\mathrm{Al} / \mathrm{C}$-filter, the solid angle of the detector, and the number of electrons used to record a spectrum, the angular yield (number of photons per electron and per unit solid angle) can be calculated. The angular yield is shown as a function of detection angle in Fig. 5(a) for titanium and in Fig. 5(b) for vanadium.

The typical Cherenkov angular profile can be clearly recognized, i.e., a symmetric profile with a minimum at zero angle and with a maximum at the angle associated with the maximum value of the refractive index. The angular profile is broadened due to small-angle elastic scattering that the electrons undergo when passing through the target foil. The divergence of the electron beam before entering the foil can be neglected. We have analyzed the influence of elastic scattering of electrons in the foil on the angular Cherenkov emission profile by performing a small-angle two-dimensional (2-D) convolution of the Cherenkov radiation angular distributions with the electron angular distribution [26]. The resulting angular photon distributions are indicated by the solid curves in Fig. 5. This broadening effect explains the nonzero angular yield value at $0^{\circ}$ and the less steep drop for higher angles. The measured angular profiles are also shifted slightly with respect to the theoretical curves, which is probably due to a small misalignment of the electron beam.

Integrating the measured angular distribution over all emission angles we find a total yield of $3.5 \times 10^{-4}$ photon/electron 
for titanium and $3.3 \times 10^{-4}$ photon/electron for vanadium. For titanium, this is slightly higher than the theoretical value of $2.4 \times 10^{-4}$ photon/electron. For vanadium, the experimental value is more than twice as high as the theoretical value of $1.4 \times 10^{-4}$ photon/electron. In view of the fact that the calculation is based on the only two refractive index data points available, i.e., the maximum value at the edge and one data point below the Cherenkov threshold, the measured spectra and angular profiles agree very well with the theoretical predictions. The fact that the yield of vanadium is higher than theoretically expected can be explained by assuming that the peak in the refractive index at the L-edge is slightly broader than according to the data of [24]. A higher peak in the refractive index data cannot explain the higher yield of vanadium, because this would give rise to a larger Cherenkov angle. The spectral resolution of the CCD camera is far too low to directly measure the actual Cherenkov radiation line width. The resulting soft $\mathrm{X}$-ray brightness and implications for source development are discussed in Section VI-B.

\section{EXPERIMENTAL RESUlTS: CARBON K-EDGE AND NICKEL L-EDGE CHERENKOV RADIATION}

\section{A. $C-K$ Cherenkov Measurements}

Carbon-K $(284 \mathrm{eV})$ Cherenkov radiation has been observed two times before, using, respectively, 1.2-GeV [11] and 75-MeV [15] electrons. We set out to demonstrate that $10-\mathrm{MeV}$ electrons should already be sufficient. In Fig. 6, the susceptibility of carbon is plotted as a function of photon energy around the $\mathrm{K}$-edge. There is only one data point that exceeds the $10-\mathrm{MeV}$ threshold, but it is sufficiently high. The angle of maximum intensity is expected at $4.2^{\circ}$. By integrating over the Cherenkov line a total yield of $4.8 \times 10^{-4}$ photon/electron is calculated. The refractive index of carbon used in these calculations is based on a density of $\rho=2.2 \mathrm{~g} / \mathrm{cm}^{3}$.

Carbon foils come in many forms with a large spread in density, which has to be taken into account because the complex susceptibility $\chi$ is proportional to the density. Several different foils have therefore been tested. Two foils have been purchased at ACF-Metals: an evaporated (amorphous) carbon foil (standard type) of $1600 \mathrm{mg} / \mathrm{cm}^{2}$ and a polycrystalline graphite foil (PCG type) of $2000 \mathrm{mg} / \mathrm{cm}^{2}$. The first foil has a density of $1.83 \pm 0.03 \mathrm{~g} / \mathrm{cm}^{3}$, which is typical for thick evaporated carbon layers, corresponding to a thickness of about $8.7 \mu \mathrm{m}$. The PCG foil is composed of graphite microcrystals and is porous. Therefore, the average density of such a foil ranges between 0.8 and $1.2 \mathrm{~g} / \mathrm{cm}^{3}$. Thus, the estimated thickness of the purchased PCG foil is about $20 \mu \mathrm{m}$, but due to the porosity it has a nonuniformity of about $40 \%$. For this foil, it is hard to predict the Cherenkov radiation output, because it is unclear which density has to be used: the density of the microcrystals (about $2.0 \mathrm{~g} / \mathrm{cm}^{3}$ ) or the average density (about $0.8-1.2 \mathrm{~g} / \mathrm{cm}^{3}$ ).

In Fig. 7(a) and (b), the spectra are shown that have been recorded at a detection angle $\Theta=4^{\circ}$ for, respectively, evaporated foil and PCG foil. Also, spectra have been recorded at other detection angles. However, no Cherenkov peak has been observed in these spectra around the carbon K-edge $(284 \mathrm{eV})$.

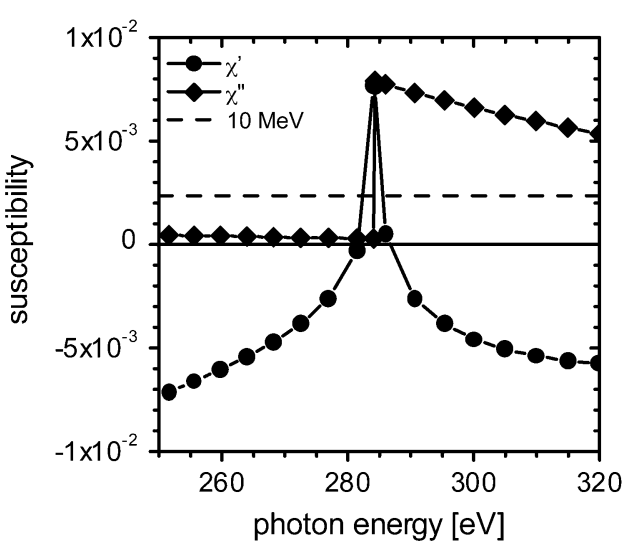

Fig. 6. Susceptibility of carbon around the K-edge $(284 \mathrm{eV})$ for $\rho=$ $2.2 \mathrm{~g} / \mathrm{cm}^{3}$. Dashed line indicates the threshold for $10-\mathrm{MeV}$ electrons.

On the basis of the carbon measurements, we conclude that the refractive index of carbon peaks less than according to [24]. A new upper limit of the refractive index can be set based on our measurements with $10-\mathrm{MeV}$ electrons: $\chi_{\max }^{\prime}=(2.4 \pm 0.3) \times$ $10^{-3}$. This conclusion is supported by independent refractive index measurements on carbon around the K-edge by Dambach et al. [27].

\section{B. Ni-L Cherenkov Measurements}

We have also tried to demonstrate that Cherenkov radiation with a photon energy higher than the water window can be generated by $10-\mathrm{MeV}$ electrons. In Fig. 8(a), the susceptibility of nickel is plotted around the L-edge $(852.7 \mathrm{eV})$. The susceptibility peaks just above the threshold value for $10-\mathrm{MeV}$ electrons. Based on these values, the maximum intensity is expected at an emission angle of about $1^{\circ}$. We have purchased a $10-\mu \mathrm{m}$-thick $25 \times 25 \mathrm{~mm}^{2}$ nickel foil (Goodfellow). We have performed a complete angular scan.

In Fig. 8(b), the recorded spectrum at $\Theta=1^{\circ}$ is plotted together with the theoretically expected spectrum. Despite the high number of frames used to obtain this spectrum, no Cherenkov radiation is observed, not even in other spectra at different detection angles. The theoretical curve shows that the Ni-L Cherenkov peak should exceed the noise level in the background transition radiation spectrum sufficiently to be detected. Since we do not observe this, it is very likely that the value of the real part of the susceptibility of nickel at the L-edge is slightly smaller than given by [24]. Independent measurements of the refractive index of nickel by Van Brug et al. [28] confirm the fact that $10-\mathrm{MeV}$ electron energy is not sufficient to generate nickel L-edge Cherenkov radiation.

\section{SOURCE DEVELOPMENT}

In Sections III and IV, it was shown that soft X-ray Cherenkov radiation can be efficiently generated with moderate electron energies, using silicon, titanium, or vanadium foils. In this section, we explore the feasibility of a compact soft X-ray source on the basis of the Cherenkov effect. The maximum brightness is obtained by sending as many electrons as possible through the smallest possible area on the target foil. In Section VI-A, we 

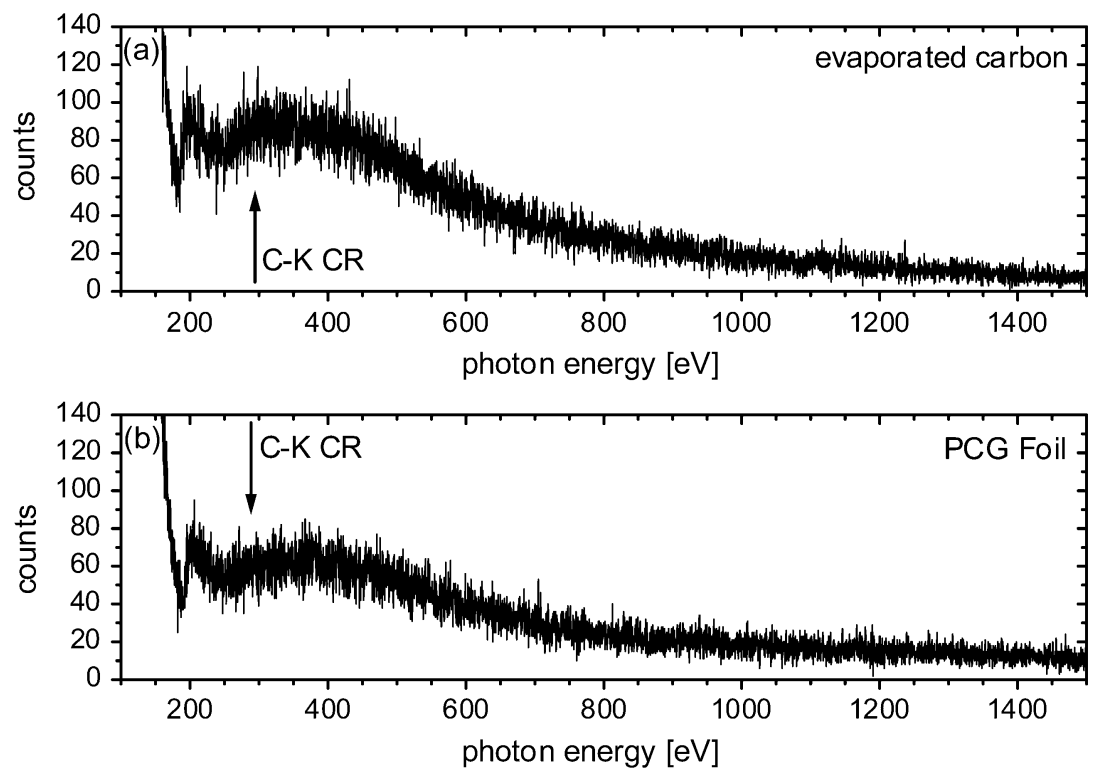

Fig. 7. CCD spectra obtained at $\Theta=4^{\circ}$ using (a) evaporated carbon and (b) polycrystalline graphite (PCG) foil. Arrows indicate the expected position of the C-K Cherenkov peak.

discuss the heat load of the electron beam on the target foil that restricts the maximum current density through the foil. Taking into account the heat load limitations, the maximum achievable brightness is presented in Section VI-B for silicon, titanium, and vanadium L-edge Cherenkov radiation. It turns out that the estimated brightness figures are comparable to the values of laser-produced plasma sources.

\section{A. Thermal Limitations}

Relativistic electrons lose a tiny fraction $\left(\approx 10^{-4}\right)$ of their kinetic energy when passing through a $\mu \mathrm{m}$-thick foil. The energy loss goes partly into Bremsstrahlung and other radiation emission effects and partly into heat. The heat deposited due to Coulomb collisions resulting in ionization and excitation of atoms is described by the collision stopping power [29], which is a function of the incident electron energy and material properties. When a high average current is sent through a foil, the total amount of energy deposited in the foil can be quite large, which may lead to significant heating of the foil. The foil may be cooled by either conduction or radiation. In practice, the melting point of the target material determines the maximum allowable temperature. In this section, estimates are made of the temperature as a function of average current and spot size of the electron beam. These two parameters limit in principle the maximum brightness that can be achieved for a soft X-ray Cherenkov source, irrespective of the accelerator performance.

Electron accelerators are pulsed electron sources with typically $2-\mu$ s pulse duration at $300-\mathrm{Hz}$ repetition rate. The heat from a single accelerator pulse is deposited in a volume determined by the electron-beam spot size and the thickness of the foil. An upper limit for the temperature rise $\Delta T$ of this volume can be estimated by neglecting the conduction and radiation cooling during the pulse and can be expressed as function of average current density $\bar{J}=f Q_{\text {pulse }} / A$ through the foil

$$
\Delta T=\frac{d E / d x}{C \rho} \frac{Q_{\text {pulse }}}{A e}=\frac{d E / d x}{C \rho} \frac{\bar{J}}{f e}
$$

with $e$ the elementary charge, $C$ the specific heat capacity, $\rho$ the mass density, $d E / d x$ energy loss per electron and per unit path length due to collisions (see Table II), $f$ the pulse repetition frequency of the accelerator, $Q_{\text {pulse }}$ the total charge per pulse, and $A$ the electron beam area. When taking the melting temperature as the limit, the maximum current density turns out to be $\bar{J} \approx 2 \mathrm{~mA} / \mathrm{mm}^{2}$ for all three Cherenkov emitting materials, assuming $f=300 \mathrm{~Hz}$.

During the time between the accelerator pulses the heat is conducted in the direction of the edge of the foil. The temperature profile in the foil $T(\mathbf{r}, t)$ can be calculated from the diffusion equation

$$
\rho C \frac{\partial T}{\partial t}=\kappa \nabla^{2} T+P_{\text {source }}-P_{\text {sink }}
$$

with $\kappa$ the heat conductivity and $P$ the power per unit volume deposited in the foil by the electron beam (source) and cooled at the edge of the foil (sink). The heat diffusion speed can be characterized by the diffusion coefficient $D$, which is defined as

$$
D=\frac{\kappa}{\rho C} .
$$

In Table II, the calculated values for the three different materials are listed. Because the target foils are very thin, the heat diffusion is two dimensional. In case the power is dumped in a very short time and the heat sink is far away from the source, the shape of the temperature profile as a function of time is described by $\exp \left(-r^{2} / 4 \pi D t\right)$. During the time between the 

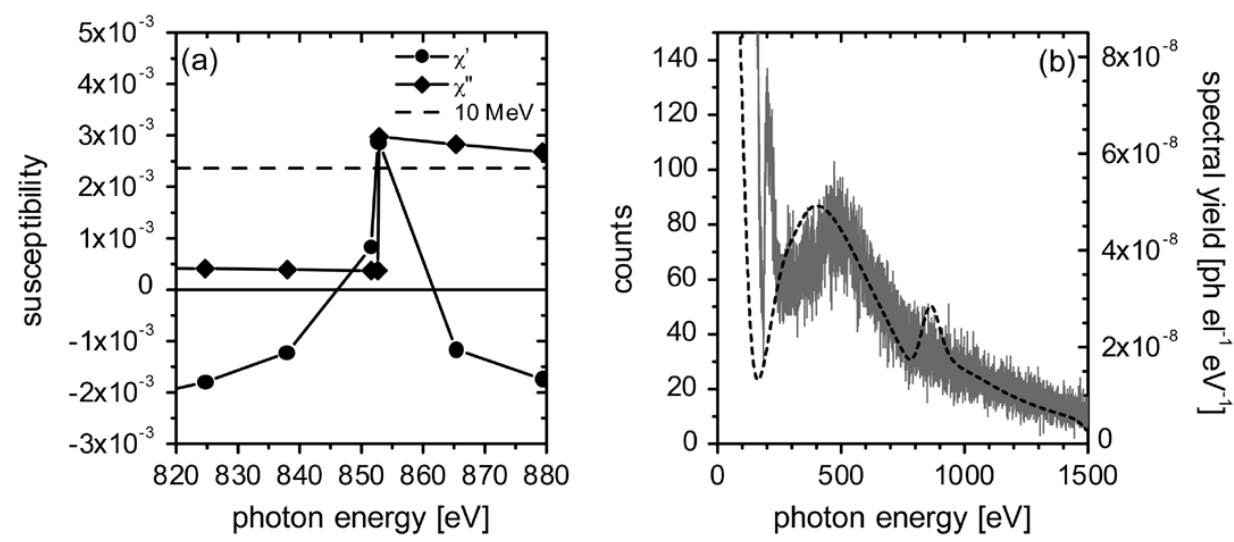

Fig. 8. (a) Susceptibility $\chi$ of nickel around the L-edge $(852.7 \mathrm{eV}$ ). Dashed line indicates the threshold for $10-\mathrm{MeV}$ electrons. (b) Pulse height spectrum of the radiation produced by $10-\mathrm{MeV}$ electrons in nickel at $\Theta=1^{\circ}$. Dashed curve is the calculated CCD spectrum.

TABLE II

MATERIAL PROPERTIES

\begin{tabular}{c|cc}
\hline Material & $\begin{array}{c}\text { Collision energy loss } \\
{\left[\mathrm{keV} \mu \mathrm{m}^{-1}\right]}\end{array}$ & $\begin{array}{c}\text { Diffusion coefficient } \\
{\left[\mathrm{cm}^{2} \mathrm{~s}^{-1}\right]}\end{array}$ \\
\hline silicon & 0.40 & $0.5-0.92$ \\
titanium & 0.68 & 0.093 \\
vanadium & 0.89 & 0.10 \\
& & \\
\hline
\end{tabular}

accelerator pulses $(f=300 \mathrm{~Hz})$, the temperature profile is typically extended by about $0.6 \mathrm{~mm}$ for the case of titanium and vanadium and about $1.4-2.0 \mathrm{~mm}$ for the case of silicon. This distance is too small to reach the boundary of the foil and thus insufficient to have it cooled down before the next accelerator pulse arrives. However, when focusing an electron beam down to a spot diameter of $0.1 \mathrm{~mm}$ or smaller, the temperature profile will diffuse out significantly between two pulses. This is of importance for cooling by radiation because the radiated power is proportional to the hot area.

We thus find that the foil cannot be cooled by conduction to the edge. Instead, the heat should be radiated from the surface of the foil. The radiated power is given by

$$
P_{\text {rad }}=\epsilon \sigma A T^{4}
$$

with $\epsilon$ the emissivity, which is a factor describing the emission efficiency compared to an ideal black body, $\sigma=5.67 \times 10^{-8}$ $\mathrm{W} \cdot \mathrm{m}^{-2} \cdot \mathrm{K}^{-4}$ the constant of Stefan-Boltzmann and $A$ the radiative area. Typically, the value of the emissivity is between 0.2 and 0.8 .

The power deposited in thermal heating due to energy loss of the electron beam should be equal to the power radiated from the front and back surface. The average current $\bar{I}$ giving rise to a temperature rise $\Delta T$ is therefore given by

$$
\bar{I}=2 \frac{\epsilon \sigma A^{*} T_{0}^{4}}{d E / d x d}\left[\left(1+\frac{\Delta T}{T_{0}}\right)^{4}-1\right]
$$

with $T_{0}$ the temperature of the surroundings, $d$ the foil thickness, and $A^{*}$ the effective black body radiation area. Assuming $A^{*} \approx 1 \mathrm{~mm}^{2}$, and taking $d=1 \mu \mathrm{m}, \epsilon=0.2$, and choosing $\Delta T$ such that $T_{0}+\Delta T$ is at $75 \%$ of the melting temperature, we find that the maximum average electron beam current that is allowed is approximately $0.02 \mathrm{~mA}$. However, the actual beam spot size may be much smaller than $1 \mathrm{~mm}^{2}$, since $A^{*}$ is determined by the thermal diffusion between pulses. For modern commercial linear accelerators a (focused) beam spot size of $0.1 \mathrm{~mm}$ is feasible.

Modern compact electron accelerators easily produce higher average currents then $0.02 \mathrm{~mA}$. To increase the average current density without damaging the target foils, the volume in which the heat is deposited may be increased by rotating the foil. High-flux rotating-anode X-ray tubes also make use of this principle. As an example, to increase the volume effectively by a factor 10, a target disk of 10-cm diameter should rotate at about $10 \mathrm{~Hz}$.

We thus conclude that by using a rotating foil, an average accelerator current of $0.2 \mathrm{~mA}$, focused on a $0.1-\mathrm{mm}$ spot size (corresponding to $\bar{J} \approx 20 \mathrm{~mA} / \mathrm{mm}^{2}$ ) is allowed without melting the foil.

\section{B. Achievable Brightness}

The maximum average current density puts an upper limit on the brightness that can be achieved with a Cherenkov source

$$
B_{\max }=\bar{J}_{\max } \frac{N}{\Delta \Omega \Delta \omega / \omega}
$$

with $\bar{J}_{\max }$ the maximum average current density, $N$ the Cherenkov yield in photons per electron, $\Delta \Omega$ the solid angle, and $\Delta \omega / \omega$ the relative spectral bandwidth of the emitted radiation. Using $\bar{J}_{\max }=20 \mathrm{~mA} / \mathrm{mm}^{2}$, the theoretical values of $\Delta \omega / \omega$, and the experimentally determined values of $N$ and $\Delta \Omega$, the values of the maximum achievable brightness for titanium, vanadium, and silicon have been calculated. The results are summarized in Table III.

The brightness values of the soft X-ray Cherenkov sources can now be compared to other sources. The typical brightness values of compact X-ray sources around $13.5 \mathrm{~nm}$ and in the water window are summarized in Tables IV and V, respectively. Of each source specific properties are specified to indicate the basis of the brightness number. The proposed soft X-ray Cherenkov source looks very promising. Cherenkov radiation is much brighter than $\mathrm{X}$-ray tubes and $\mathrm{HH}$ sources. In the 
TABLE III

SPECTRAL BRIGHTNESS CALCULATION BASED ON THE EXPERIMENTAL PARAMETERS (YIELD, $\theta_{\min }, \theta_{\text {max }}$ ) AND THEORETICAL FWHM-BANDWIDTH $\Delta \omega / \omega$ AND Assuming a 0.2-mA Average CurRent 10-MeV Electron ACCElERATOR With 0.1-mm ElECtron-BEAm Spot Size

\begin{tabular}{c|ccccc}
\hline material & $\begin{array}{c}\text { Cherenkov yield } \\
\times 10^{-4}[\mathrm{ph} / \mathrm{el}]\end{array}$ & $\begin{array}{c}\Delta \omega / \omega \\
\times 10^{-3}\end{array}$ & $\begin{array}{c}\theta_{\min } \\
\text { [degrees] }\end{array}$ & $\begin{array}{c}\theta_{\max } \\
\text { [degrees] }\end{array}$ & $\begin{array}{c}\text { spectral brightness } \\
{\left[\mathrm{ph} \cdot \mathrm{s}^{-1} \cdot \mathrm{mm}^{-2} \cdot \mathrm{mrad}^{-2} \cdot 0.1 \% \mathrm{BW}^{-1}\right]}\end{array}$ \\
\hline $\mathrm{Ti}$ & 3.5 & 4.4 & 0.0 & 4.7 & $0.6 \times 10^{9}$ \\
$\mathrm{~V}$ & 3.3 & 2.3 & 0.0 & 5.0 & $1.0 \times 10^{9}$ \\
$\mathrm{Si}$ & 8.0 & 10 & 5 & 12 & $1.1 \times 10^{8}$
\end{tabular}

TABLE IV

SPECTRAL BRIGHTNESS $\left[\mathrm{PH} \cdot \mathrm{s}^{-1} \cdot \mathrm{mm}^{-2} \cdot \mathrm{mrad}^{-2} \cdot 0.1 \% \mathrm{BW}^{-1}\right]$ COMPARISON OF COMPACT X-RAY SOURCES AROUND $13.5 \mathrm{~nm}$

\begin{tabular}{|c|c|c|c|c|c|c|}
\hline source type & $\begin{array}{l}\text { photon energy } \\
{[\mathrm{eV}]}\end{array}$ & energy source & $\begin{array}{l}\text { relative } \\
\text { bandwidth } \\
{[0.1 \%]}\end{array}$ & $\begin{array}{l}\text { solid angle } \\
\text { [sr] }\end{array}$ & $\begin{array}{l}\text { source } \\
\text { size } \\
{[\mathrm{mm}]}\end{array}$ & $\begin{array}{l}\text { spectral } \\
\text { brightness }\end{array}$ \\
\hline $\begin{array}{l}\text { Z-pinch } \\
\text { plasma [30] }\end{array}$ & $\begin{array}{l}91.9 \mathrm{eV}(13.5 \mathrm{~nm}) \\
\text { with } \mathrm{Xe}\end{array}$ & 120-Hz discharge & 20 & $4 \pi$ & 0.5 & $1.5 \times 10^{10}$ \\
\hline $\begin{array}{l}\text { Cherenkov } \\
\text { source }\end{array}$ & $\begin{array}{l}99.7 \mathrm{eV}(12.4 \mathrm{~nm}) \\
\text { from } \mathrm{Si}-\mathrm{L}\end{array}$ & $\begin{array}{l}10-\mathrm{MeV}, \quad 0.2-\mathrm{mA} \\
\text { electron beam }\end{array}$ & 10 & 0.11 & 0.1 & $1.1 \times 10^{8}$ \\
\hline $\begin{array}{l}\text { EUV tube } \\
{[2]}\end{array}$ & $\begin{array}{l}91.9 \mathrm{eV}(13.5 \mathrm{~nm}) \\
\text { from silicon }\end{array}$ & $\begin{array}{l}\text { Continuous } 10- \\
\mathrm{keV}, 1-\mathrm{mA} \text { electron } \\
\text { beam }\end{array}$ & 20 & $2 \pi$ & 0.1 & $1.2 \times 10^{6}$ \\
\hline
\end{tabular}

TABLE V

SPECTRAL BRIGHTNESS $\left[\mathrm{PH} \cdot \mathrm{s}^{-1} \cdot \mathrm{mm}^{-2} \cdot \mathrm{mrad}^{-2} \cdot 0.1 \% \mathrm{BW}^{-1}\right.$ ] COMPARISON OF COMPACT X-RAY SOURCES IN THE WATER WINDOW

\begin{tabular}{|c|c|c|c|c|c|c|}
\hline source type & $\begin{array}{l}\text { photon energy } \\
{[\mathrm{eV}]}\end{array}$ & energy source & $\begin{array}{l}\text { relative } \\
\text { bandwidth } \\
{[0.1 \%]}\end{array}$ & $\begin{array}{l}\text { solid angle } \\
{[\mathrm{sr}]}\end{array}$ & $\begin{array}{l}\text { source } \\
\text { size } \\
{[\mathrm{mm}]}\end{array}$ & $\begin{array}{l}\text { spectral } \\
\text { brightness }\end{array}$ \\
\hline $\begin{array}{l}\text { Laser } \\
\text { Produced } \\
\text { Plasma [8] }\end{array}$ & $\begin{array}{l}368 \text { eV (3.37 } \mathrm{nm}) \\
\mathrm{C}_{\mathrm{IV}} \text { from liquid } \\
\text { ethanol }\end{array}$ & $\begin{array}{l}\text { 100-Hz, } 10-\text { Watt } \\
\text { Nd:YAG laser }\end{array}$ & 3.3 & $4 \pi$ & 0.025 & $6 \times 10^{9}$ \\
\hline $\begin{array}{l}\text { Cherenkov } \\
\text { source }\end{array}$ & $\left.\begin{array}{llll}512 & \mathrm{eV} & (2.42 & \mathrm{nm}\end{array}\right)$ & $\begin{array}{l}\text { 10-MeV, } \quad 0.2-\mathrm{mA} \\
\text { electron beam }\end{array}$ & 2.4 & 0.024 & 0.1 & $1 \times 10^{9}$ \\
\hline $\begin{array}{l}\text { High- } \\
\text { Harmonic } \\
\text { generation } \\
{[7]}\end{array}$ & $\begin{array}{l}284 \mathrm{eV}(4.37 \mathrm{~nm}) \text { in } \\
\text { helium }\end{array}$ & $\begin{array}{l}\text { 1-kHz Ti:sapphire } \\
\text { laser }\end{array}$ & $\begin{array}{l}\text { continuous in } \\
\text { water window }\end{array}$ & $\begin{array}{l}\text { well- } \\
\text { collimated }\end{array}$ & - & $5 \times 10^{7}$ \\
\hline $\begin{array}{l}\text { Liquid } x \text {-ray } \\
\text { tube [3] }\end{array}$ & $\begin{array}{l}525 \mathrm{eV}(2.36 \mathrm{~nm}) \\
\text { from oxygen }\end{array}$ & $\begin{array}{l}10-\mathrm{keV}, \quad 60-\mu \mathrm{A} \\
\text { electron beam on } \\
\text { water jet }\end{array}$ & 7.2 & $2 \pi$ & 0.1 & $7 \times 10^{7}$ \\
\hline
\end{tabular}

water window, the brightness of the soft X-ray Cherenkov source using vanadium is in the same order of magnitude as the brightness achieved with laser-produced plasma sources.

\section{Applications: Soft X-Ray Microscopy and X-Ray Photoelectron Spectroscopy}

The most challenging imaging application is soft X-ray microscopy, requiring a high brightness source. Soft X-ray microscopy has been demonstrated using a compact laser-produced plasma source [8] with a brightness between $10^{9}$ and $10^{10} \mathrm{ph} \cdot \mathrm{s}^{-1} \cdot \mathrm{mm}^{-2} \cdot \mathrm{mrad}^{-2} \cdot 0.1 \% \mathrm{BW}^{-1}$. Using a linear accelerator, which is operated at $0.2-\mathrm{mA}$ average current, with the electron beam focused to a spot size of $0.1 \mathrm{~mm}$ on a vanadium foil, the expected Cherenkov source brightness is about
$1 \times 10^{9} \mathrm{ph} \cdot \mathrm{s}^{-1} \cdot \mathrm{mm}^{-2} \cdot \mathrm{mrad}^{-2} \cdot 0.1 \% \mathrm{BW}^{-1}$, comparing favorably with the laser-produced plasma source.

Probably the best way to realize soft X-ray microscopy with a Cherenkov source is to use an off-axis ellipsoidal multilayer mirror under grazing incidence to illuminate the sample and subsequently image the sample on a CCD camera using a zone plate lens. As an illustration, we may put in some concrete numbers [31].

We start with either a Ti-L or a V-L Cherenkov source driven by a $10-\mathrm{MeV} 0.2-\mathrm{mA}$ average current electron beam, focused to a $0.1-\mathrm{mm}$ spot. A total flux of approximately $4 \times 10^{11}$ photons/s is therefore emitted in the angular profiles shown in Fig. 5. About $50 \%$ of the emitted radiation, i.e., the radiation emitted at angles smaller than $4^{\circ}$, is captured by an off-axis ellipsoidal $\mathrm{Ni}$-Ti multilayer mirror. The ellipsoidal mirror reflects about 
$35 \%$ of the captured part of the Cherenkov cone under an average grazing angle of $10^{\circ}$ and focuses it onto the sample. The condensor mirror images the $0.1-\mathrm{mm}$ source spot without any (de)magnification onto the sample, which typically has a size of about $10 \mu \mathrm{m}$, so only about $1 \%$ of the reflected light impinges on the sample. Assuming 10- $\mu \mathrm{m}$ thickness, the watery parts of the sample will transmit approximately $25 \%$, which is then available for imaging. A zone plate with a $50-\mu \mathrm{m}$ diameter and a minimum zone width of $30 \mathrm{~nm}$, which is placed at a distance of $0.5 \mathrm{~mm}$ behind the sample, subsequently images the sample, magnified approximately $700 \times$, onto a CCD camera at $35 \mathrm{~cm}$ behind the sample. A typical $20 \times 20 \mu \mathrm{m}$ pixel on the CCD chip thus corresponds to the $30-\mathrm{nm}$ spatial resolution of the objective lens. The half-opening angle of the zone plate lens is slightly more than $3^{\circ}$, i.e., well matched to the light cone illuminating the sample, which is the reason we chose not to have any demagnification in the condensing stage. Since the efficiency of the zone plate lens is about $10 \%$ and the sample is imaged onto approximately $10^{5}$ pixels, approximately $1 \times 10^{2}$ photons per second impinge on a pixel for the watery parts of the sample. Using a back-illuminated CCD camera with a quantum efficiency of about $65 \%$, this implies that in this setup exposure times of the order of $10 \mathrm{~s}$, corresponding to $\sim 10^{3}$ electron-hole pairs per pixel, are sufficient to obtain the required high contrast.

A second interesting application is X-ray photoelectron spectroscopy. In this case, the flux on the target position is specified, which is a derived value from the brightness without a firm requirement for bandwidth and solid angle. Typically, the flux has to be larger than $10^{8} \mathrm{ph} / \mathrm{s}$ on a $10-\mu \mathrm{m}$ spot. The flux required for a practical exposure time in X-ray microscopy, calculated above, also amply fulfills the requirements for X-ray photoelectron spectroscopy.

\section{REFERENCES}

[1] C. Jacobsen, "Soft X-ray microscopy," Trends Cell Biol., vol. 9, pp. 44-47, 1999.

[2] A. Egbert, B. Mader, B. Tkachenko, A. Ostendorf, C. Fallnich, B. N. Chichkov, T. Missalla, M. C. Schürmann, K. Gäbel, G. Schriever, and U. Stamm, "Compact electron-based extreme ultraviolet source at 13.5 nm," J. Microlith., Microfab., Microsyst., vol. 2, pp. 136-139, 2003.

[3] B. Buijsse, "A keV-electron-based tabletop soft X-ray source," in Proc. SPIE, vol. 4502, 2001, pp. 74-81.

[4] L. Malmqvist, L. Rymell, M. Berglund, and H. M. Hertz, "Liquid-jet target for laser-plasma soft X-ray generation," Rev. Sci. Instrum., vol. 67, pp. 4150-4153, 1996.

[5] R. Lebert, G. Schriever, T. Wilhein, and B. Niemann, "Soft X-ray emission of laser-produced plasmas using a low-debris cryogenic nitrogen target," J. Appl. Phys., vol. 84, pp. 3419-3421, 1998.

[6] C. H. Spielmann, N. H. Burnett, S. Sartania, R. Koppitisch, M. Schnürer, C. Kan, M. Lenzner, P. Wobrauschek, and F. Krausz, "Generation of coherent X-rays in the water window using 5-femtosecond laser pulses," Science, vol. 278, pp. 661-664, 1997.

[7] E. A. Gibson, A. Paul, N. Wagner, R. Tobey, D. Gaudiosi, S. Backus, I. P. Christiv, A. Aquila, E. M. Gullikson, D. T. Attwood, M. M. Murnana, and H. C. Kapteyn, "Coherent soft X-ray generation in the water window with quasiphase matching," Science, vol. 302, pp. 95-98, 2003.

[8] G. A. Johansson, A. Holmberg, H. M. Hertz, and M. Berglund, "Design and performance of a laser-plasma-based compact soft X-ray microscope," Rev. Sci. Instrum., vol. 73, pp. 1193-1197, 2002.

[9] M. Wieland, R. Frueke, T. Wilhein, C. Spielmann, M. Pohl, and U. Kleineberg, "Submicron extreme ultraviolet imaging using high-harmonic radiation," Appl. Phys. Lett., vol. 81, pp. 2520-2522, 2002.
[10] P. Rullhusen, X. Artru, and P. Dhez, Novel Radiation Sources Using Relativistic Electrons: From Infrared to X-rays, Singapore: World Scientific, 1998

[11] V. A. Bazylev, V. I. Glebov, E. I. Denisov, N. K. Zhevago, M. A. Kumakhov, A. S. Khlebnikov, and V. G. Tsinoev, "X-ray Cerenkov radiation. Theory and experiment," Sov. Phys. JETP, vol. 54, pp. 884-892, 1981.

[12] W. Knulst, O. J. Luiten, M. J. van der Wiel, and J. Verhoeven, “Observation of narrow-band Si L-edge Cerenkov radiation generated by $5 \mathrm{MeV}$ electrons," Appl. Phys. Lett., vol. 79, pp. 2999-3001, 2001.

[13] W. Knulst, M. J. van der Wiel, O. J. Luiten, and J. Verhoeven, "Highbrightness, narrowband, and compact soft X-ray Cherenkov sources in the water window," Appl. Phys. Lett., vol. 83, pp. 4050-4052, 2003.

[14] C. Grupen, Particle Detectors. Cambridge, MA: Cambridge Univ. Press, 1996.

[15] M. J. Moran, B. Chang, M. B. Schneider, and X. K. Maruyama, "Grazing-incidence Cherenkov X-ray generation," Nucl. Instrum. Methods, vol. B48, p. 287, 1990.

[16] A. E. Kaplan, C. T. Law, and P. L. Shkolnikov, "X-ray narrow-line transition radiation source based on low-energy electron beams traversing a multilayer nanostructure," Phys. Rev., vol. E52, pp. 6795-6808, 1995.

[17] B. Lastdrager, A. Tip, and J. Verhoeven, "Theory of Čerenkov and transition radiation from layered structures," Phys. Rev., vol. E61, pp. 5767-5778, 2000.

[18] M. L. Ter-Mikaelian, High-Energy Electromagnetic Processes in Condensed Media. New York: Wiley, 1972, ch. 4.

[19] I. M. Frank and I. E. Tamm, "Coherent visible radiation of fast electrons passing through matter," Dokl. Akad. Nauk. USSR, vol. 14, pp. 109-114, 1937.

[20] V. L. Ginzburg and I. M. Frank, Zh. Eksp. Teor. Fiz., vol. 16, pp. 15-9, 1946.

[21] V. L. Ginzburg and V. N. Tsytovich, Transition Radiation and Transition Scattering, Bristol: Hilger, 1990, ch. 2.

[22] J. V. Jelley, Cherenkov Radiation and Its Applications. London: Pergamon, 1958.

[23] W. Knulst, Cherenkov Radiation in the Soft X-ray Region: Toward a Compact Narrowband Source. Eindhoven, The Netherlands: Universiteitsdrukkerij TU/e, 2004.

[24] B. L. Henke, E. M. Gullikson, and J. C. Davis. (1993) X-ray interactions: Photoabsorption, scattering, transmission, and reflection at $\mathrm{E}=$ 50-30000 eV, Z = 1-92. At. Data Nucl. Data Tables [Online], vol (2), pp. 181-342. Available: http://www-cxro.lbl.gov

[25] J. W. den Herder et al., "The reflection grating spectrometer on board XMM-Newton," Astron. Astrophys., vol. 365, pp. L7-L17, 2001.

[26] J. Corstens, W. Knulst, O. J. Luiten, and M. J. van der Wiel, An efficient method for calculating plural scattering of relativistic electrons in thin foils and multilayers, in Nucl. Instrum. Methods, vol. B, 2004. accepted for publication.

[27] S. Dambach, H. Backe, Th. Doerk, N. Eftekhari, H. Euteneuer, F. Görgen, F. Hagenbuck, K. H. Kaiser, O. Kettig, G. Kube, W. Lauth, H. Schöpe, A. Steinhof, Th. Tonn, and Th. Walcher, "Novel interferometer in the soft X-ray region," Phys. Rev. Lett., vol. 80, p. 5473, 1998.

[28] H. van Burg, M. P. de Bruijn, R. van der Pol, and M. J. van der Wiel, "Ni-C multilayer reflectivity and photoelectron yield in the $\mathrm{Ni}_{L}$-edge region," Appl. Phys. Lett., vol. 49, p. 914, 1986.

[29] M. J. Berger, J. S. Coursey, and M. A. Zucker. Stopping-power and range tables for electrons, protons, and helium ions. Physical Reference Data [Online]. Available: http://www.physics.nist.gov/PhysRefData/Star/Text/contents.html

[30] R. Stuik, "Characrerization of XUV Sources," Ph.D. dissertation, Eindhoven Univ. Technology, 2002.

[31] M. Reijnders, N. Kleemans, P. Jonkers, and W. Aarden, "Compact soft x-ray microscopy with Cherenkov radiation," Eindhoven Univ. Technology, Internal Rep., 2004.

Walter Knulst was born in Alkmaar, The Netherlands, in 1976. He received the M.Sc. degree in engineering physics from Twente University of Technology, Twente, in 1999, and the Ph.D. degree from Eindhoven University of Technology, Eindhoven, The Netherlands, in 2004.

From 1999 to 2003, he worked as a Ph.D. student at the Department of Applied Physics, University of Eindhoven, on the subject of soft X-ray Cherenkov radiation. Since 2004, he has been a Postdoctoral Researcher at Delft University of Technology, Delft, The Netherlands, where he investigates charge carrier dynamics in polymeric semiconductors using the technique of pulse radiolysis. 
Jom Luiten was born in Eindhoven, The Netherlands, in 1961. He received the M.Sc. degree in engineering physics at Eindhoven University of Technology, Eindhoven, in 1987, and the Ph.D. degree in experimental physics at the University of Amsterdam in 1993.

From 1994 to 1996, he worked at the Space Research Organization, Utrecht, The Netherlands, on the development of superconducting tunnel junction X-ray detectors. In 1996 and 1997, he was with ASML, working on the development of wafer stepper lithography machines. Since 1997, he has been an Assistant Professor for the Department of Applied Physics, Eindhoven University of Technology, where he works on the physics and applications of compact high-brightness electron accelerators.
Jan Verhoeven was born in The Hague, The Netherlands, in 1942. In 1970, he graduated from Delft University of Technology, Delft, The Netherlands.

Since 1970, he has been with the FOM Institute for Atomic and Molecular Physics, Amsterdam, The Netherlands. Until 1980, he was the Leader of the Vacuum Physics Group. From 1980 to 2001, he was the Leader of the Thin Film Group. He is specialized in the onset of layer growth and the influence of the impact of energetic ions. He has successfully applied this in the formation of multilayer systems for X-ray reflection and optical applications. Since 2001, he has been the leader of the Nano Fabrication Group. His present research interests include the growth of periodic arrays of metallic clusters on oxide surfaces and their interaction with light. 